\title{
A kind of multimode nonlinear algorithm for efficiency-improved course keeping of large tankers
}

\author{
Hongbiao Zhao ${ }^{1}$, and Xianku Zhang ${ }^{1, *}$ \\ ${ }^{1}$ Navigation College, Dalian Maritime University, Dalian, China
}

Keywords: Course keeping, Efficiency-improved control, Nonlinear feedback, Multimode algorithm.

\begin{abstract}
In order to achieve efficient control of ship course keeping with low energy consumption, this paper introduces nonlinear feedback into fuzzy control and closed-loop gain shaping algorithm (CGSA). Taking the ship "New Triumph" as an example, the simulation results show that the multimode controller with non-linear feedback can not only respond to the steering instructions quickly, but also reduce the energy consumption by $32 \%$ to realize the course keeping efficiently and smoothly under the wind-wave model. Research shows that the introduction of nonlinear feedback term can optimize the control system, and its advantages of saving energy consumption are of great significance in practical engineering applications.
\end{abstract}

\section{Introduction}

With the continuous development of the world economy, the increasingly large-scale ship has become an inevitable trend. In ship motion control field, the ship is a large inertia system, especially for large oil tanker. When a certain rudder angle is steered, the response of ship attitude adjustment is slow even consuming dozens or hundreds of seconds. Therefore, for a certain course in the course of steering, how to achieve energy-efficient, safe and stable course maintenance is always a hot issue in this field.

With the continuous development of intelligent control and classical control algorithms and the higher and higher requirements of course keeping control, it is difficult to achieve multiple improvements of control performance in all aspects only by a single control algorithm. In recent years, the author and his research team had carried out a lot of research on advanced ship motion control strategies [1-3]. In addition, in recent three years, the application of neural network in ship course keeping nonlinear control was given in reference [4]; the application of variable universe adaptive fuzzy control in marine main engine speed control system was given in reference [5]; nonlinear feedback control was

\footnotetext{
* Corresponding author: hongbiao0522@,163.com
} 
applied to ANFIS in reference [6], which was achieved more quickly and accurately of ship's course keeping; the nonlinear feedback theory was successfully applied to the course keeping control of surface unmanned vehicle in reference [7]. Based on these researches, this paper combines concise robust control with fuzzy control $[8,9]$, uses non-linear feedback to deal with the input of the controller, and carries out simulation analysis for course keeping of large oil tanker.

\section{Mathematical model of ship motion}

A response-based non-linear mathematical model of ship motion is adopted for the design of a simple robust controller, the controlled plant is taken as the responsive nonlinear mathematical model of ship motion

$$
\ddot{\psi}+\frac{K_{0}}{T_{0}}\left(\alpha \dot{\psi}+\beta \dot{\psi}^{3}\right)=\frac{K_{0}}{T_{0}} \delta
$$

In Equation(1): $\psi$ is the heading angle, $\delta$ is the rudder angle, $K_{0}, T_{0}$ are the manoeuvrability indices of the ship, $\alpha, \beta$ are the proportional coefficients of yaw rate $\dot{\psi}$.If the nonlinear term is ignored, the Notomo transfer function model of the controlled plant can be obtained

$$
G_{\psi \delta}(s)=K_{0} /\left(s\left(T_{0} s+1\right)\right)
$$

Formula (2) is applied to the design of a concise robust controller.

Norrbin nonlinear mathematical model of ship motion is used for ship model in numerical simulation $[10,11]$. Establishing a six-degree-of-freedom nonlinear model for the simulation of ship motion closed-loop control system, which is

$$
\begin{gathered}
\dot{X}_{(4)}=A_{(4)} X_{(4)}+B_{(4)} \delta_{r}+\left\{\begin{array}{c}
\left(I_{(2)}^{\prime}{ }^{-1}\right)\left[\mathrm{F}_{\mathrm{NON}}^{\prime}+\mathrm{F}_{\mathrm{WIND}}^{\prime}+\mathrm{F}_{\mathrm{WAVE}}^{\prime}\right] \\
0 \\
0
\end{array}\right\} \\
\dot{x}_{0}=u \cos \psi-v \sin \psi \\
\dot{y}_{0}=u \sin \psi-v \cos \psi
\end{gathered}
$$

where $\boldsymbol{I}_{(2)}^{\prime}$ is the inertial force derivative matrix of the two-degree-of-freedom state-space ship linear mathematical model, $\mathrm{F}_{\mathrm{NON}}^{\prime}, \mathrm{F}_{\mathrm{WIND}}^{\prime}, \mathrm{F}_{\mathrm{WAVE}}^{\prime}$ are the nonlinear hydrodynamic, wind and wave terms, respectively. $\left[\boldsymbol{x}_{\boldsymbol{0}}, \boldsymbol{y}_{\boldsymbol{0}}\right]^{\mathrm{T}}$ is used to solve the ship position, $u, v$ are the surge speed and sway speed of the ship. In this paper, the Norrbin model is used for the nonlinear fluid force in equation (3), which is

$$
\mathrm{F}_{\mathrm{NON}}^{\prime}=\left[\begin{array}{l}
\boldsymbol{Y}_{N O N}^{\prime} \\
\boldsymbol{N}_{N O N}^{\prime}
\end{array}\right]=\left[\begin{array}{l}
f_{Y}(v, r) \\
C f_{N}(v, r)
\end{array}\right]
$$


in equation (4),

$$
\begin{aligned}
f_{Y}(v, r)=\left\{\begin{array}{lr}
d_{M} \cdot r|r|\left[-\frac{1}{12}-\frac{1}{L^{2}}\left(\frac{v}{r}\right)^{2}\right] & -\infty<-\frac{1}{L} \frac{v}{r}<-\frac{1}{2} \\
d_{M} \cdot r|r|\left[-\frac{1}{2} \frac{1}{L} \frac{v}{r}-\frac{2}{3} \frac{1}{L^{3}}\left(\frac{v}{r}\right)^{3}\right] & -\frac{1}{2} \leq-\frac{1}{L} \frac{v}{r} \leq \frac{1}{2} \\
d_{M} \cdot r|r|\left[\frac{1}{12}+\frac{1}{L^{2}}\left(\frac{v}{r}\right)^{2}\right] & \frac{1}{2}<-\frac{1}{L} \frac{v}{r}<\infty
\end{array}\right. \\
f_{N}(v, r)=\left\{\begin{array}{lr}
d_{M} \cdot r|r|\left[-\frac{1}{6} \frac{1}{L}\left(\frac{v}{r}\right)\right] & -\infty<-\frac{1}{L} \frac{v}{r}<-\frac{1}{2} \\
d_{M} \cdot r|r|\left[-\frac{1}{32}-\frac{1}{4} \frac{1}{L^{2}}\left(\frac{v}{r}\right)^{2}+\frac{1}{6} \frac{1}{L^{4}}\left(\frac{v}{r}\right)^{4}\right] & -\frac{1}{2} \leq-\frac{1}{L} \frac{v}{r} \leq \frac{1}{2} \\
d_{M} \cdot r|r|\left[\frac{1}{6} \frac{1}{L}\left(\frac{v}{r}\right)\right] & \frac{1}{2}<-\frac{1}{L} \frac{v}{r}<\infty
\end{array}\right.
\end{aligned}
$$

The proportional coefficient $C$ in equation (4) is the dimensionless crossflow coefficient, $d_{M}$ in equation (5) is the draft of midship, $L$ is the length between the two perpendiculars of the ship, and $r$ is the angular velocity of the ship. The wind term $\mathrm{F}_{\text {WIND }}^{\prime}$ is divided into average wind and fluctuating wind. For average wind and fluctuating wind, the calculation programs are written into the ship model according to reference [12]. For wave force term $\mathrm{F}_{\mathrm{WAVE}}^{\prime}$, a linear wave model is used to replace the complex nonlinear wave model. A linear approximation of International Towing Tank Conference (ITTC) spectral density function can be written

$$
y(s)=h(s) \omega(s)
$$

where $\omega(s)$ is a zero-mean Gaussian white noise process with a power spectrum of $G_{\omega \omega}(\omega)=1.0$ and $h(s)$ is a second-order transfer function.

$$
h(s)=\frac{K_{\omega} s}{s^{2}+2 \zeta \omega_{0} s+\omega_{0}^{2}}
$$

$K_{\omega}$ is defined as $K_{\omega}=2 \zeta \omega_{0} \sigma_{\omega}$, where $\sigma_{\omega}$ is the constant describing wave strength, $\zeta$ is the damping coefficient, and $\omega_{2}$ is the dominant wave frequency. In ITTC spectrum, $\omega_{2}=\frac{4.85}{T_{\omega}}, \sigma_{\omega}{ }^{2}=0.0185 T_{\omega} h_{1 / 3}^{2}, T_{\omega}$ is the wave period and $h_{1 / 3}$ is the significant wave height. Taking $T_{\omega}=8 \mathrm{~s}, h_{1 / 3}=8 \mathrm{~m}, \zeta=0.3$, the second-order wave transfer function corresponding to Beaufort No.6 is obtained.

$$
h(s)=\frac{0.4198 s}{s^{2}+0.3637 s+0.3675}
$$




\section{Multimode nonlinear control system}

The structure diagram of multimode fuzzy nonlinear control system is shown in Fig. 1[13]. In this paper, a combination of fuzzy controller and simple robust controller is adopted. By segmenting the variable universe, fuzzy control is adopted in the domain that needs to improve the response speed and damping performance of the system. Near the equilibrium point, we hope to reduce the steady state error, eliminate small oscillation and adopt a simple robust controller. At the same time, the input of the controller is processed nonlinearly by using the non-linear feedback technology, which makes the system controller output with less energy consumption.

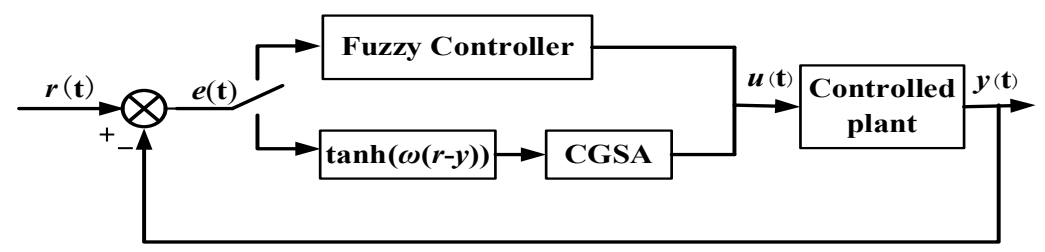

Fig. 1. The structure diagram of multimode fuzzy nonlinear control system.

\subsection{Design of fuzzy controller}

The structure of the fuzzy controller is shown in Fig. 2.

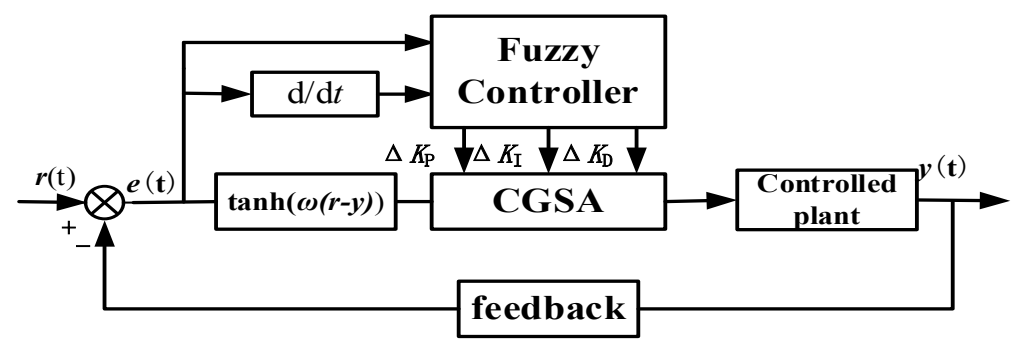

Fig. 2. The structure of the fuzzy controller.

The fuzzy controller takes heading error e and heading error changing rate ec as input values, and adjustments $\Delta K_{\mathrm{P}}, \Delta K_{\mathrm{I}}$ and $\Delta K_{\mathrm{D}}$ of parameters of closed-loop gain shaping algorithm (CGSA) as output values. Fuzzy linguistic variables are $\{\mathrm{NB}, \mathrm{NM}, \mathrm{NS}, \mathrm{ZE}, \mathrm{PS}, \mathrm{PM}, \mathrm{PB}\}$. The input and output of fuzzy reasoning are divided by triangular membership function. For the range of values of fuzzy variables, all the input and output ranges in this paper are $[-6,6]$. According to the specific physical meaning and function of the control parameters, combined with field debugging and expert operation experience, the fuzzy control rules are shown in Fig. 3. 

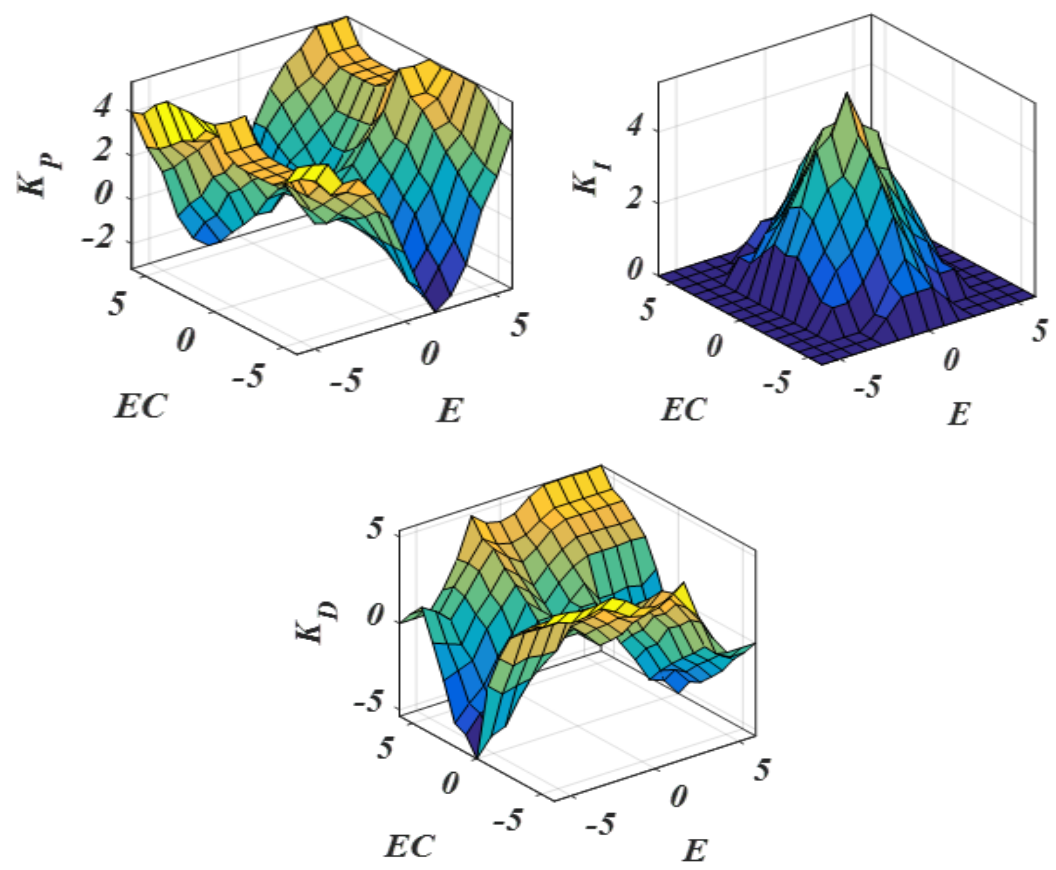

(a)

(b)

(c)

Fig. 3. Fuzzy control rules.

\subsection{Design of concise robust controller}

Enlightened by the loop shaping theory, a control algorithm based on closed-loop gain shaping is presented through survey of the singular value curve of $\mathrm{H} \infty S / T$ complex sensitivity as well as the correlativity of $S$ and $T(T=I-S)$. Observing the typical S\&T singular value curve shown in Fig. 4, in order to make the system robust and stable, the closed-loop spectrum of the system is required to be low-pass, and the maximum singular value is 1 to ensure that the reference signal is tracked with zero steady-state error. The bandwidth frequency of the system determines the control performance of the system; and the high frequency asymptote slope determines the sensitivity of the system to interference outside the effective frequency band. The larger the slope, the less sensitive the system is to interference. The stronger the robustness of the system, the higher the order of the designed controller, which is not conducive to the realization of the controller and the control effect has not been significantly improved. In this paper, the high frequency asymptote slope of the concise robust controller is chosen to be $-20 \mathrm{~dB} / \mathrm{dec}$. 


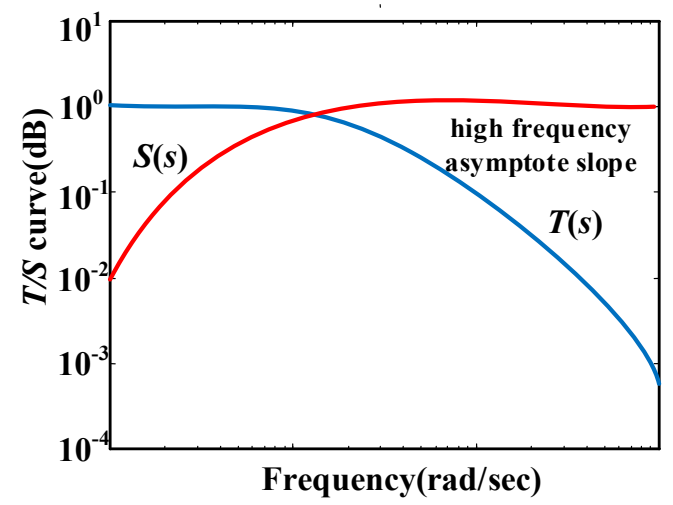

Fig. 4. Typical S\&T singular value curve.

Fig. 5 is a standard feedback structure, $\mathrm{K}$ is a controller and $\mathrm{G}$ is a controlled object.

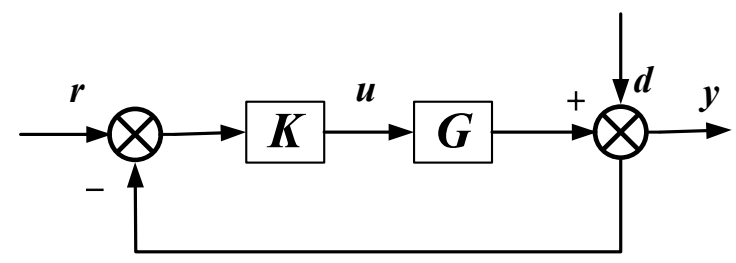

Fig. 5. Standard feedback structure.

According to the CGSA, the frequency spectrum of the closed-loop system is made equal to the frequency spectrum of a first-order inertial system with the largest singular value approximately one, then

$$
\frac{1}{T_{1} \mathrm{~s}+1}=\frac{G K}{1+G K}, K=\frac{1}{G T_{1} \mathrm{~s}}
$$

To eliminate the steady state error using the closed loop gain shaping algorithm, a minor constant $\varepsilon$ is added into the denominator of Nomoto model, then the Notomo model is obtained

$$
G(s)=\frac{K_{0}}{T_{0} s^{2}+s+\varepsilon}
$$

Substituting (10) into (9),

$$
K(s)=\frac{1}{K_{0} T_{1}}+\frac{\varepsilon}{K_{0} T_{1}} \frac{1}{s}+\frac{T_{0}}{K_{0} T_{1}} s
$$

Considering the large inertia of ship motion, the dynamic performance of course keeping control system for ships can be improved greatly when a positive constant $\rho$ is added to the proportional part of the control law Equation(11). The corresponding theoretical analysis and simulation test are given in reference[14].Then, the controller is obtained 


$$
K=\frac{1}{K_{0} T_{1}}+\rho+\frac{\varepsilon}{K_{0} T_{1}} \frac{1}{s}+\frac{T_{0}}{K_{0} T_{1}} s
$$

\section{Numerical simulations and result analysis}

Wind and wave disturbances in Numerical simulation see Chapter, Mathematical Model of Ship Motion. In order to be more accordance with the reality of navigation, the course keeping control system with pure delay, dead-zone, hysteresis, saturation and other nonlinear characteristics is approximated as a first-order inertia component in consideration of the maximum rudder angle and steering speed, and according to the analysis of actual marine test steering data, the steering gear system is limited to operate a rudder as fast as 6 seconds.

The numerical simulations are carried out in Simulink, the solver is ode 45 and solver's type is variable-step. Taking the "New Triumph" oil tanker as an example, the ship parameters under full loaded are shown in Table 1 . Where $B$ is the beam of ship, $X_{\mathrm{C}}$ is the distance from centre of gravity to the midship, $A_{R}$ is rudder area, $V$ is ship speed, $\nabla$ is displacement, $C_{b}$ is the block coefficient of ship.

Table 1. Particulars of "New Triumph".

\begin{tabular}{|c|c|}
\hline Particulars & Full loaded \\
\hline$L[\mathrm{~m}]$ & 327 \\
\hline$B[\mathrm{~m}]$ & 60 \\
\hline$X_{\mathrm{C}}[\mathrm{m}]$ & 10.07 \\
\hline$A_{R}\left[\mathrm{~m}^{2}\right]$ & 128 \\
\hline${ }_{2}[\mathrm{kn}]$ & 15.7 \\
\hline$\nabla\left[\mathrm{m}^{3}\right]$ & 368786 \\
\hline$d_{M}[\mathrm{~m}]$ & 22.5 \\
\hline$C_{b}$ & 0.8024 \\
\hline
\end{tabular}

Set the bandwidth frequency $1 / T_{1}\left(1 / T_{1}\right.$ should be crossover frequency in the strict sense) of the two closed systems to $0.10(\mathrm{rad} / \mathrm{s})$ and $0.33(\mathrm{rad} / \mathrm{s})$, respectively. For Notomo model, 0.00001 for minor constant of denominator $\varepsilon$ and 3 for positive constant $\rho$ to improve control performance. The nonlinear feedback parameter $\omega=0.20$. In the numerical simulation, the angle threshold of heading error is chosen as 5 degrees (that is, when heading error is greater than 5 degrees, nonlinear fuzzy control is adopted, and when heading error is less than 5 degrees, nonlinear concise robust control is adopted).

Assuming that the numerical simulation is carried out under the condition that the ship has a setting course of $60^{\circ}$ and a wind direction of $40^{\circ}$, a comparison is made between the multimode nonlinear efficiency-improved controller and the concise robust controller designed by using the closed-loop gain algorithm alone. The simulation block diagram and simulation results are shown in Fig. 6 and Fig. 7. 


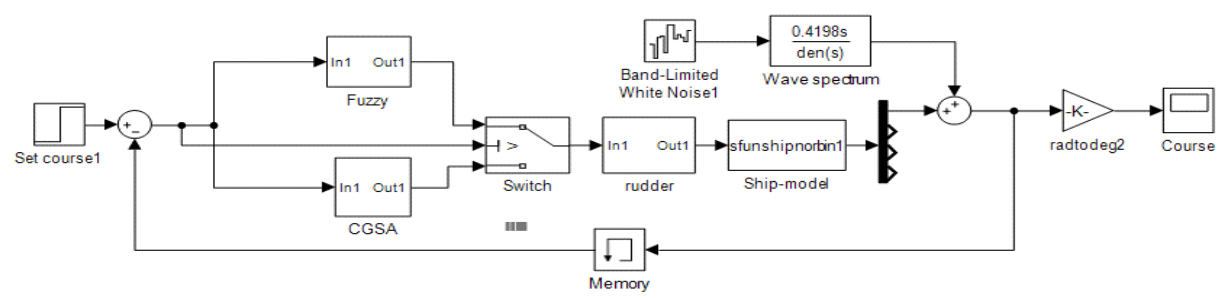

Fig. 6. Simulation block diagram.

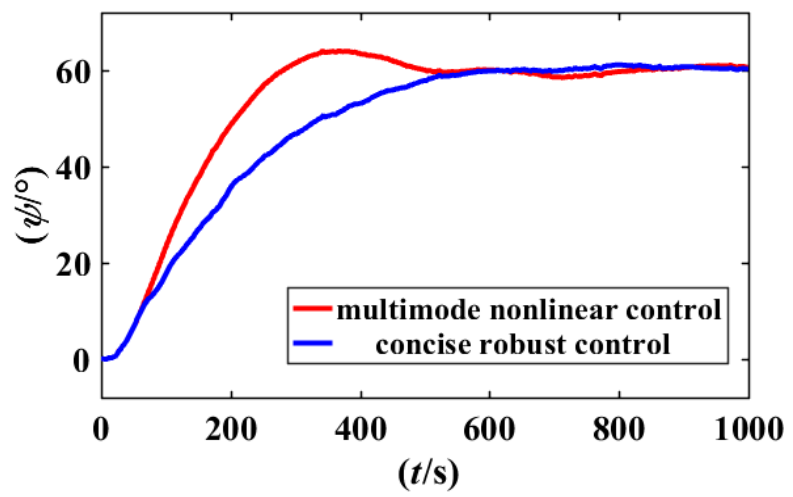

(a)

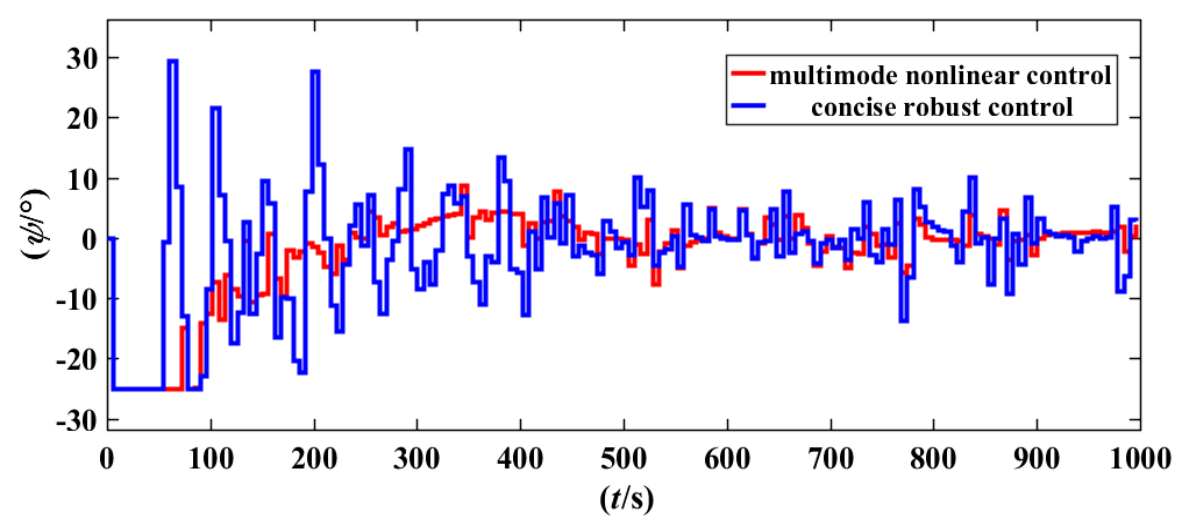

(b)

Fig. 7. Simulation results.

From the comparison of simulation results in Fig. 7, it can be seen that although the time of course stabilization of multimode nonlinear control is approximately the same as that of concise robust control, under the condition of very small overshoot, the response speed of ship course is obviously improved by multimode nonlinear control driven by the fuzzy system, and the first time to reach the setting course shortened nearly 200 seconds, which has certain significance for the emergency maneuvering of ships to avoid danger. In order to better compare the two algorithms, absolute error mean MAE and average rudder angle MIC are introduced to characterize the performance of the system in course keeping and energy consumption. 


$$
\begin{aligned}
& \mathrm{MAE}=\frac{1}{t_{s}-t_{0}} \int_{t_{0}}^{t_{s}}\left|\psi_{r}-\psi(t)\right| \mathrm{d} t \\
& \mathrm{MIC}=\frac{1}{t_{s}-t_{0}} \int_{t_{0}}^{t_{s}}|\delta(t)| \mathrm{d} t
\end{aligned}
$$

The numerical simulation results are shown in Table 2.

Table 2. Numerical simulation results.

\begin{tabular}{|c|c|c|}
\hline & $\begin{array}{c}\mathrm{MAE}\left({ }^{\circ}\right) \\
(\text { Simulation time }=300 \mathrm{~s})\end{array}$ & $\begin{array}{c}\mathrm{MIC}\left({ }^{\circ}\right) \\
(\text { Simulation time }=550 \mathrm{~s})\end{array}$ \\
\hline $\begin{array}{c}\text { Multimode Nonlinear } \\
\text { Control }\end{array}$ & 11.9176 & 0.2634 \\
\hline Concise Robust Control & 17.5092 & 0.3827 \\
\hline
\end{tabular}

In order to avoid serious damage to the ship's attitude caused by steering at large angles and thus affect navigation safety, the maximum steering rudder angle is limited to 25 degrees. From Table 2, we can see that the nonlinear multimode control not only has a slight advantage in heading stability difference, but also reduces the average rudder angle from $17.5092^{\circ}$ to $11.9176^{\circ}$, and the average rudder angle drops by $32 \%$. Therefore, the non-linear feedback technology can greatly reduce the energy consumption in the process of ship operation, which is for shipping companies. It is of great significance. In addition, the controller design in this paper only uses the linear Notomo model, while the simulation uses the nonlinear Norrbin model with the nonlinear characteristic limitation of the steering servo system, that is equivalent to the model perturbation, and the course keeping control effect is still satisfactory, which shows that the designed controller has good robustness.

\section{Conclusion}

In order to design a controller with better control effect, this paper introduces non-linear feedback into fuzzy control and closed-loop gain shaping algorithm. This paper takes the "New Triumph" as an example to carry out the Numerical simulations. The results show that the multimode controller with nonlinear feedback still has good control effect under the wind-wave model. In the whole simulation process, the ship's course can not only respond to the steering instructions quickly, but also maintain the course safely and efficiently with a small average steering angle. It protects the steering gear and saves energy. Although the simulations in this paper show that the algorithm can save energy consumption to achieve fast response of ship course keeping, at the same time, it also brings some overshoots of ship course. Many physical rules also show that fast and accurate control process is often difficult to achieve in most cases, but how to make a better balance between response speed and control accuracy is still the direction of further research in the future.

Much appreciations to each reviewer for their valuable comments and suggestions to improve the quality of this note. This work is partially supported by the National Science Foundation of China (Grant No.51679024 and No.51909018), the Fundamental Research Funds for the Central University (Grant No.3132016315), and the University 111 Project of China (Grant No.B08046). The authors would like to thank anonymous reviewers for their valuable comments to improve the quality of this article. 


\section{References}

1. Z. Xianku, Control Algorithm for Autopilot Driven by Sine of Course Deviation. Navigation of China, 2011,34(1):1-4.

2. Z. Guoqing, Z. Xianku. Concise robust adaptive path-following control of underactuated ships using DSC and MLP. IEEE Journal on Oceanic Engineering, 2014, 39(4):685-694.

3. Z, Xianku, Z. Guoqing, Determining robust parameters in stabilizing set of Backstepping based nonlinear controller for ships' course keeping. Transactions of the Royal Institution of Naval Architects Part A: International Journal of Maritime Engineering. 158(4):269-273.

4. S. Na, Q. Meihong, Application of the PBF neural network in nonlinear control of ship course keeping. SHIP SCIENCE AND TECHNOLOGY, 2017, 39(10): 94-96.

5. W. Lijun, W. Sisi Concise Robust Control of Marine Engine Speed based on Backstepping and its Fuzzy Comprehension. Complexity, vol. 2019.

6. Z. Zhiheng, Z. Xianku, Z. Guoqing ANFIS-based course-keeping control for ships using nonlinear feedback technique Journal of Marine Science and Technology,2018.

7. F. Yunsheng, M. Dongdong, Z. Xianku. et al. Course keeping Control Based on Integrated Nonlinear Feedback for a USV with Pod-like Propulsion.Journal of Navigation,2018,71(4):1-21.

8. L. Hongxing, W. Jiayin, M. Zhihong, Modeling of Fuzzy Control System. SCIENCE IN CHINA, 2002(09).

9. Z. Naiyao, Structure Analysis of Typical Fuzzy Controller. Fuzzy System and Mathematics, 1997, 11(2): 10 21.

10. N. H. Norrbin, Theory and Observations on the Use of a Mathematical Model for Ship Manoeuvring in Deep and Confined Waters. SSPA, Publication, 1971,No. 68, Gothenburg.

11. N. H Norrbin, Further studies of parameter identification of linear and nonlinear ship steering dynamics. SSPA, Gothenburg, Sweden. Report 1977,1920-6.

12. Z. Xianku, J. Yicheng, Control System Modeling and Digital Simulation. Dalian Maritime University Press, 2013.

13. Z. Dongjian, M. Ning, G. Xiechong, Adaptive Fuzzy Compensation Control for Nonlinear Ship Course Keeping. Shanghai Jiaotong Univ.(Sci.), 2015, 49(02):250-254.

14. Z. Xian-ku, G. Wei .Improved Concise Robust Control of Course Keeping for Ships With Large inertia .Navigation of China , 2010, 33(3):1-5. 